\title{
Cosmic Ray Composition in the Second Knee Region as Measured by the TALE Hybrid Detector
}

\section{Keitaro Fujita ${ }^{a, *}$ on behalf of the Telescope Array Collaboration}

(a complete list of authors can be found at the end of the proceedings)

${ }^{a}$ Graduate School of Science, Osaka City University,

3-3-138, Sugimoto, Sumiyoshi-ku, Osaka, 558-8585, Japan

E-mail: mvpxic0130@cosmicray-ocu.jp

The Telescope Array Low-energy Extension (TALE) experiment is a hybrid air shower detector for the observation of air showers induced by cosmic rays with energy above $10^{16} \mathrm{eV}$. The TALE detector consists of a Fluorescence Detector (FD) station with 10 FD telescopes located at the TA Middle Drum FD Station (itself made up of 14 FD telescopes), and a Surface Detector (SD) array made up of 80 scintillation counters, including 40 with $400 \mathrm{~m}$ spacing and 40 with $600 \mathrm{~m}$ spacing. A triggering system for the TALE-SD using an external trigger from the TALE-FD, a so-called hybrid trigger, allows for a lower energy threshold. The TALE hybrid trigger system has been working since 2018. Here we present an estimate of the performance of hybrid detection using a Monte Carlo simulation, and a first measurement of the cosmic ray composition and spectrum using the TALE-Hybrid detector.

$37^{\text {th }}$ International Cosmic Ray Conference (ICRC 2021)

July 12 th - 23rd, 2021

Online-Berlin, Germany

\footnotetext{
${ }^{*}$ Presenter
} 


\section{Introduction}

The Telescope Array (TA) cosmic ray observatory is the largest hybrid cosmic ray detector in the northern hemisphere designed to detect ultra high energy cosmic rays. The main part of the experiment consists of a surface detector (SD) array that is overlooked by three fluorescence detector (FD) stations. TA SD consists of 507 scintillation counters with $1200 \mathrm{~m}$ spacing and covering a total of $\sim 700 \mathrm{~km}^{2}$ area on the ground. The three TA FD stations are Black Rock Mesa (BRM), Long Ridge (LR), and Middle Drum(MD). These TA FD telescopes are viewing $3^{\circ}$ to $31^{\circ}$ in elevation.

The Telescope Array Low-energy Extension(TALE), located at the north part of the TA Experiment site, is aimed at measuring the energy spectrum and the mass composition of very high energy cosmic rays above $10^{16} \mathrm{eV}$. The TALE detector consists of one FD station with ten fluorescence telescopes and an array of 80 scintillation surface detectors, which were deployed to cover a total area of approximately $20 \mathrm{~km}^{2}$. The TALE-FD began operation in 2013 at the MD station. All 10 telescopes were refurbished from components previously used by HiRes [1], and view $31^{\circ}$ to $59^{\circ}$ in elevation, directly above the field of view of the MD telescopes. The TALE telescopes are instrumented with FADC electronics. The TALE-SD consists of 40 scintillation counters with 400 $\mathrm{m}$ and 40 counters with $600 \mathrm{~m}$ spacing, and started observation from 2017. In addition, an external trigger from the TALE-FD to the TALE-SD to detect low energy cosmic rays, so-called hybrid trigger system, was installed in 2018. The TALE detector configuration is shown in Fig. 1. The full details of the detectors are found in [2] [3] .
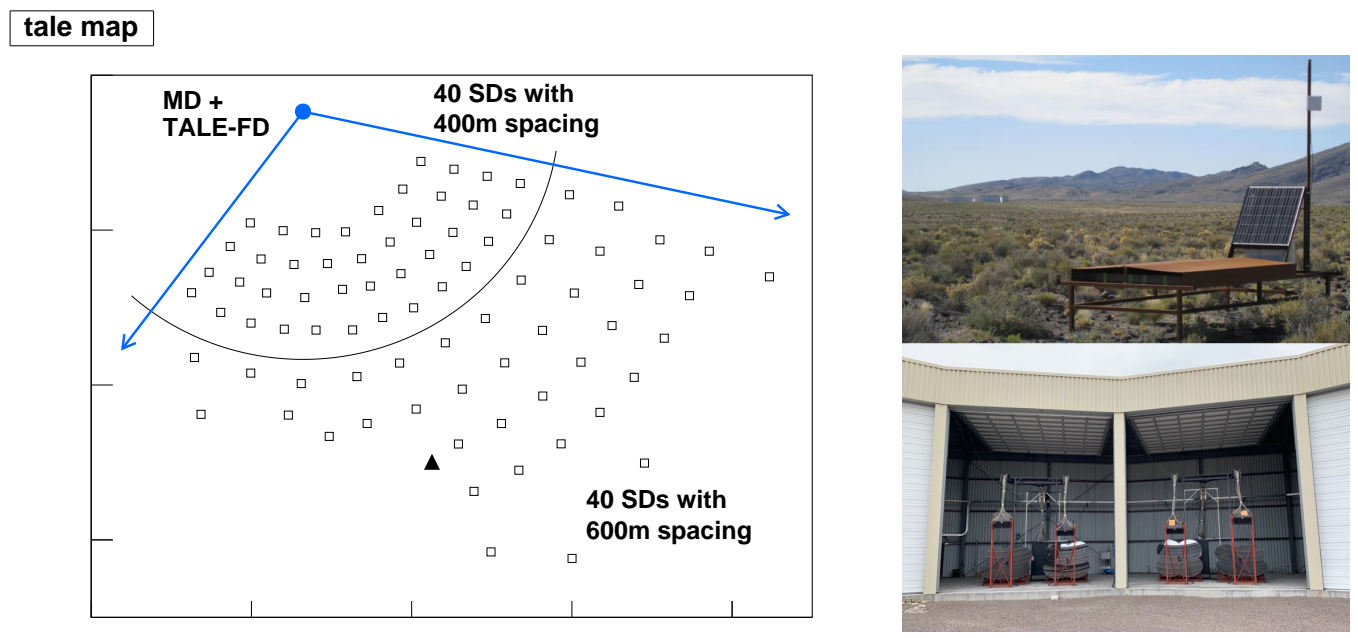

Figure 1: Left: The layout of the TALE detector. Open square boxes represent the locations of the TALE SD counters and small filled circle correspond to the MD / TALE FD station. The arrows represent azimuthal viewing ranges of both FDs. Top-Right: A deployed SD in the field. Bottom-Right: The TALE telescopes.

\section{Event Reconstruction}

The event reconstruction procedure consists of the following main steps: First, the showerdetector plane (SDP) is reconstructed from the pattern and pointing direction of the triggered PMT 
pixels.

Next, we apply Profile-Constrained Geometry Fit (PCGF) reconstruction [4] which simultaneously fit the shower geometry and the shower profile. We successfully reconstructed low energy events by PCGF in the monocular analysis [3], and we use it also here. The shower geometry is calculated by the time vs angle fit which uses the pointing directions and timings of the PMTs. The expected timing of $i$-th PMT is

$$
t_{\text {exp }, i}=t_{\text {core }}+\frac{1}{c} \frac{\sin \psi-\sin \alpha_{i}}{\sin \left(\psi+\alpha_{i}\right)} r_{\text {core }}
$$

where $t_{\exp , i}$ and $\alpha_{i}$ are the expected timing and elevation angle in the SDP for the $i$-th PMT, respectively, $t_{\text {core }}$ is the timing when the air shower reached the ground, $r_{\text {core }}$ is the distance from the FD station to the shower core, and $\psi$ is at an shower inclination angle in the SDP. For an event that has the timing information of one SD near the shower core, $t_{\text {core }}$ is expressed by

$$
t_{\text {core }}=t_{\mathrm{SD}}+\frac{1}{c}\left(r_{\text {core }}-r_{\mathrm{SD}}\right) \cos \psi
$$

where $t_{\mathrm{SD}}$ is the timing of the leading edge of the SD signal. This is an advantage of the hybrid reconstruction technique. The two observable, $t_{\mathrm{SD}}$ and $r_{\mathrm{SD}}$, are added to the relation of the eq(1), and as a result the number of the fitting parameter is reduced to two and the geometry determination accuracy is improved. The quantity to be minimized in the fitting is written as

$$
\chi^{2}=\sum_{i} \frac{\left(t_{\mathrm{exp}, i}-t_{i}\right)^{2}}{\sigma_{\mathrm{t}, i}^{2}}
$$

where $\sigma_{\mathrm{t}, i}$ is the fluctuation of the signal timing. Once the shower geometry is determined, the shower profile is fitted using the Gaisser-Hillas parameterization formula [5]

$$
N(x)=N_{\max }\left(\frac{x-X_{0}}{X_{\max }-X_{0}}\right)^{\frac{X_{\max }-X_{0}}{\lambda}} \exp \left(\frac{X_{\max }-x}{\lambda}\right)
$$

where $N(x)$ is the number of charged particles at a given slant depth, $x, X_{\max }$ is the depth of shower maximum, $N_{\max }$ is the maximum number of particles at $X_{\max }, X_{0}$ is the depth of the first interaction, and $\lambda$ is interaction length of shower particles.

Two examples of the data event triggered by TALE Hybrid detector are shown in Fig. 2 and Fig. 3. Fig. 2 is typical one telescope cherenkov dominated event observed by the TALE detector. Fig. 3 is high energy event observed by MD and TALE detectors. In the later event, the shower profile reconstruction was done using both MD and TALE PMT signals.

\section{Monte Carlo Simulation and Data / MC Comparison}

The performance of our detectors and the reconstruction programs are evaluated using our Monte Carlo program. The TALE MC package consists of two parts, those are the air shower generation part and the detector simulation part. We generate cosmic ray showers using the CORSIKA-based MC simulation code developed for TA [6]. Here we use proton and iron primary particles with QGSJETII-04 [7] hadronic interaction model. The generated MC follow a broken 

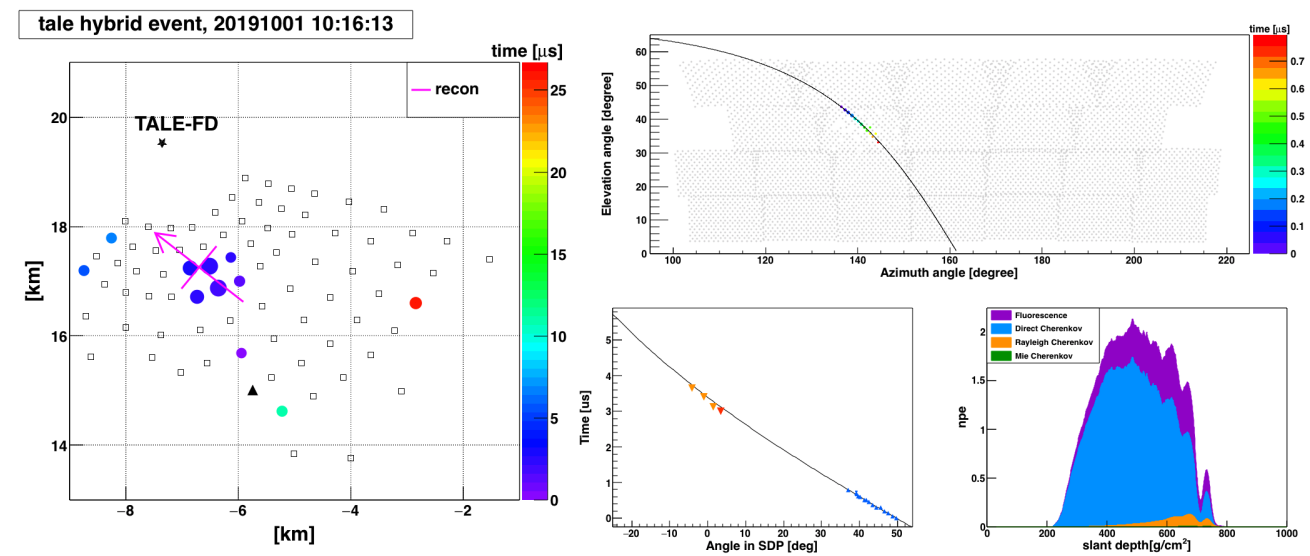

Figure 2: One-telescope low energy cherenkov event. Left: SD display. Reconstructed shower direction is indicated by magenta arrow and crossed point is the reconstructed shower core position. Top-Right: FD display. Bottom-Middle: Time vs angle fit. The blue triangle makers are FD PMT timing and inverted triangle are SD timing. The red inverted triangle is a detector which is used in eq(2). Bottom-Right: Reconstructed shower profile with relative contributions of fluorescence light, cherenkov light and scattered cherenkov light.
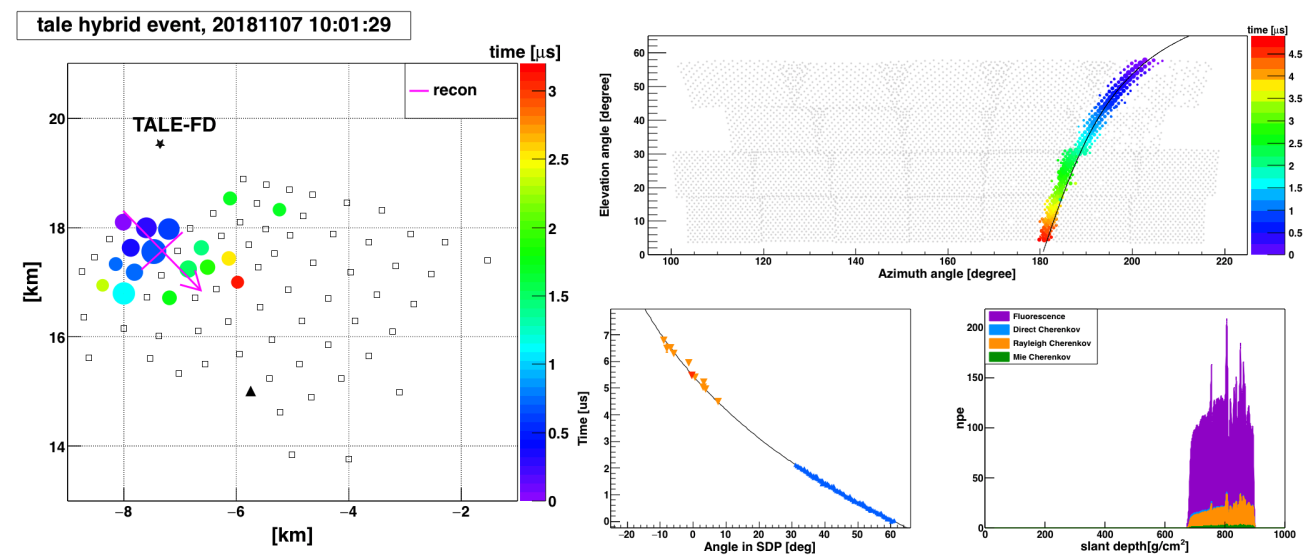

Figure 3: High energy fluorescence event. Left: SD display. Top-Right: FD display. Bottom-Middle: Time vs angle fit. Bottom-Right: Reconstructed shower profile.

power low spectrum which spectrum index is -2.9 below $10^{17.1} \mathrm{eV}$ and is -3.2 above $10^{17.1} \mathrm{eV}$. All of the calibration factors with time dependence are applied to SD and FD detector simulations.

Fluorescence events dominate at energies above $10^{17.5} \mathrm{eV}$, and cherenkov events dominate at below $10^{17} \mathrm{eV}$. Fluorescence and cherenkov events have very different characteristics, so that different event selection criteria were applied for the different types of events, as summarized in Table 1, in order to remove poorly reconstructed events and ensure good detector resolution. Here we define fluorescence events as fractional contribution to the total signal of Fluorescence Light (FL) $>0.75$, and cherenkov events as fractional contribution to the total signal of FL $\leq 0.75$. Fig. 4 shows the resolution of the important shower parameters. We obtain the resolutions of $\sim 3 \%$ in $R_{p}, \sim 1^{\circ}$ in $\psi$ angle, $\sim 30 \mathrm{~g} / \mathrm{cm}^{2}$ in $X_{\max }$ and $\sim 10 \%$ in energy $\left(E \geq 10^{16.5} \mathrm{eV}\right)$. In addition, Data/MC comparisons were performed with TALE-Hybrid events to show that our MC describes 
the observed events well as shown in Fig. 5. The data and MC were applied the same quality cuts to be compared.

\begin{tabular}{llll}
\hline \hline Variable & CL & & FL \\
\hline No saturated PMTs in FD & & $\begin{array}{l}\text { applied } \\
\text { applied }\end{array}$ & \\
SD detected $\geq 3$ MIPs & & applied & \\
$X_{\text {max bracketing cut }}$ & track $>6.5^{\circ}$ & & \\
Angular track-length [deg] & $>100 \mathrm{~ns}$ & & \\
Event duration [ns] & $>10$ & & $>2000$ \\
\# of PMTs & $>50$ & \\
\# of Photo-electrons / \# of PMTs & & \\
\# of Photo-electrons & & \\
\hline
\end{tabular}

Table 1: Quality Cuts Applied in this study
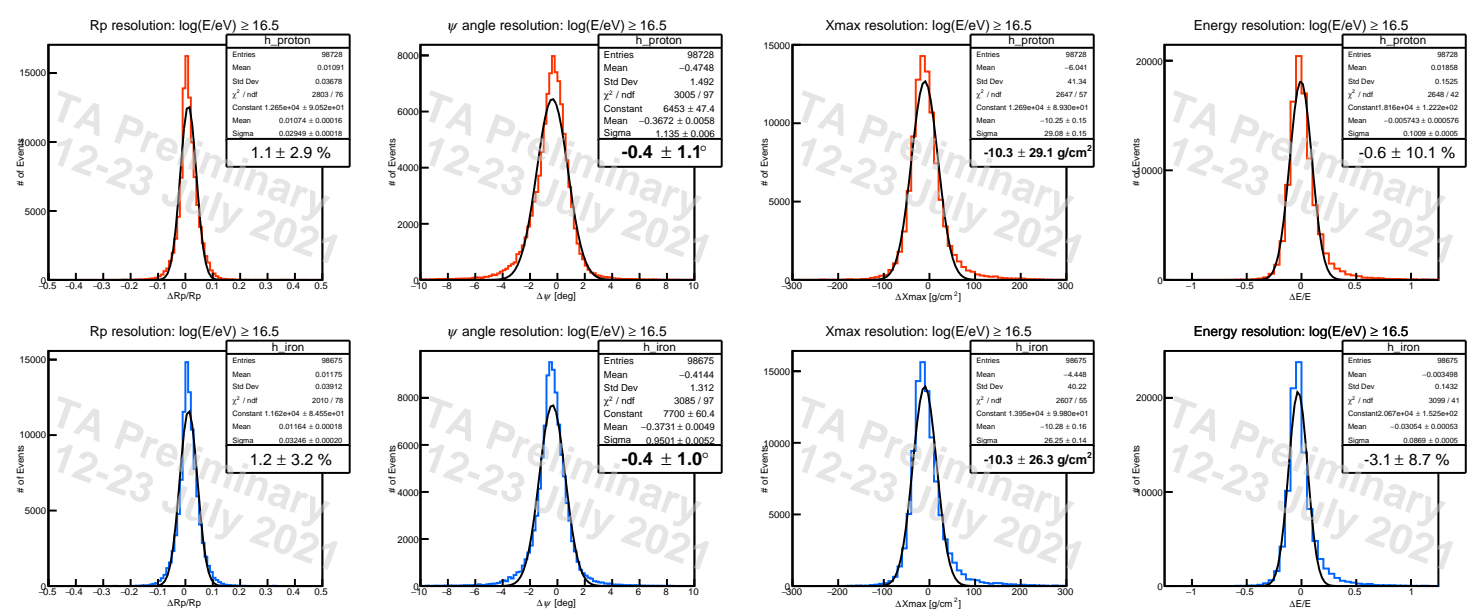

Figure 4: Reconstruction resolutions of the impact parameter $R_{p}$, the shower inclination angle in the SDP $\psi$, the shower maximum $X_{\max }$, and the shower energy $E$ respectively. Top panels are shown for proton MC case and bottom panels are shown for iron MC case. The black curve is a Gaussian fit to the distribution of the uncertainty.

\section{Composition Analysis}

The TALE Hybrid data collected between November 2017 and February 2021 is included in the analysis. The total detector on-time in this period is $\sim 980$ hours. We presented the results of a measurement of the cosmic rays composition in the energy range of $10^{16.6}-10^{18.4} \mathrm{eV}$. Fig. 6a shows $X_{\max }$ distributions divided by 4 energy ranges. The black points with error bar are measured $X_{\max }$, the red distribution is pure proton MC prediction and the blue distribution is pure iron MC prediction. Reconstructed TALE Hybrid events mean $X_{\max }$ as a function of shower energy is shown in Fig. 6b. Reconstructed $X_{\max }$ values for 2 MC primaries shown alongside the data for comparison. We also show mean $X_{\max }$ measured by the main TA Hybrid detector [8] and HiRes/MIA [9]. 

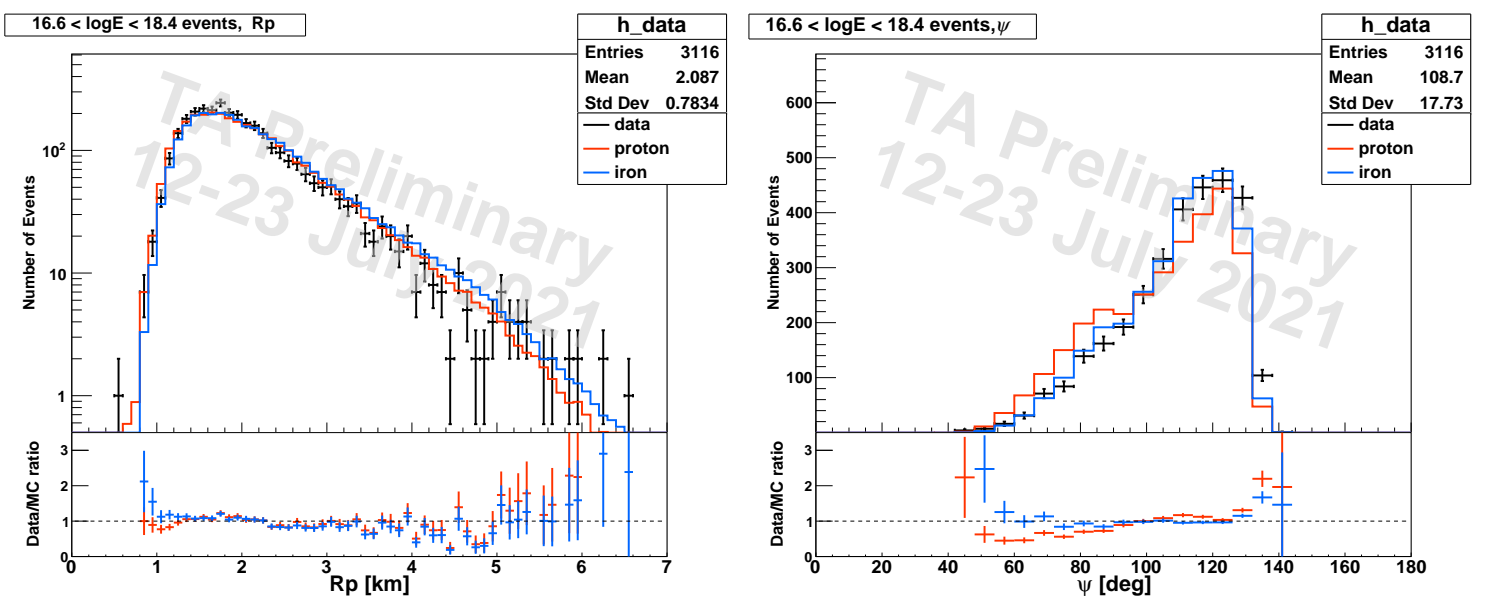

Figure 5: Data / MC comparisons. From left to right, the impact parameter Rp, the shower inclination angle in the SDP $\psi$, respectively. The black points with error bars show the data, while the proton/iron MC is shown by the red/blue histogram. The MC has been normalized to the same number of events as the data.
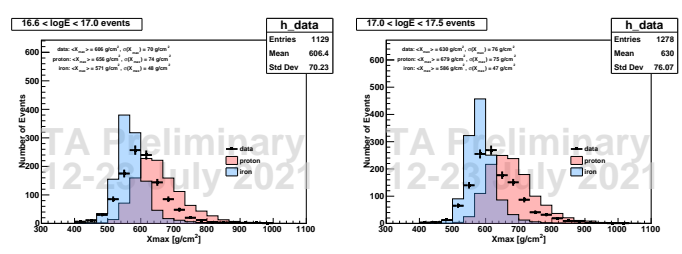
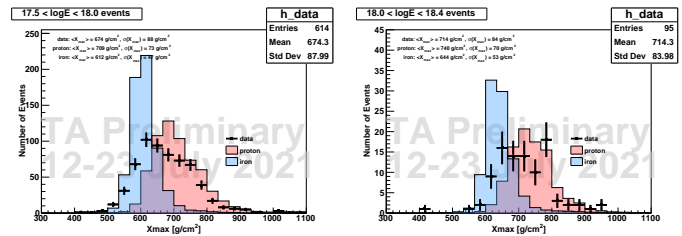

(a)

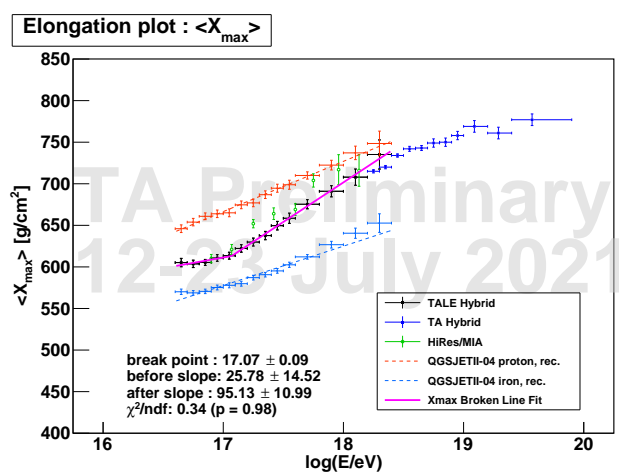

(b)

Figure 6: (a): $X_{\max }$ distributions compared with the expected distributions estimated by pure component MC. (b): Mean $X_{\max }$ as a function of shower energy, measured by TALE Hybrid detector.

\section{Energy Spectrum}

To evaluate the energy spectrum, it is essential to calculate an aperture and an exposure of the Hybrid detector. The aperture cannot calculate a simple geometrical factor because it depends on not only the energies, but also the performance of FD, atmospheric models, PMT gains and primary particles. Thus, we estimate the aperture of our detector using MC simulations including these dependences. The aperture is calculated by

$$
A \Omega(E)=A \Omega_{\text {gen }} \cdot N_{\text {recon }}(E) / N_{\text {thrown }}(E)
$$

where $E$ is the primary energy of cosmic ray, $\mathrm{A} \Omega_{\mathrm{gen}}$ is the thrown aperture region of $\mathrm{MC}$ simulation, $N_{\text {recon }}$ is the number of reconstructed events and $N_{\text {thrown }}$ is the number of thrown events. Then we obtain the exposure to multiply the aperture by the total on-time. Fig. 7a shows estimated exposure 
with proton, iron and H4a [10] composition assumption. The bottom panel of Fig. 7a shows the relative differences in the exposure to proton, iron and $\mathrm{H} 4 \mathrm{a}$ assumption with respect to the $50 \%$ proton $+50 \%$ iron mixture. The proton and iron points are shifted slightly to allow for greater visibility. Knowing the exposure, we calculate a preliminary energy spectrum. The energy spectrum is evaluated by the aperture assuming the H4a composition. The missing energy also estimated using by H4a composition assumption. It is shown in Fig. $7 \mathrm{~b}$ with the latest measurements by TA.

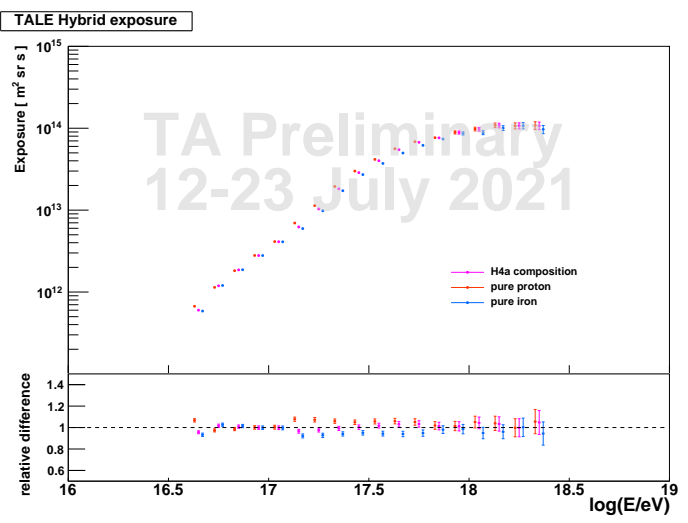

(a)

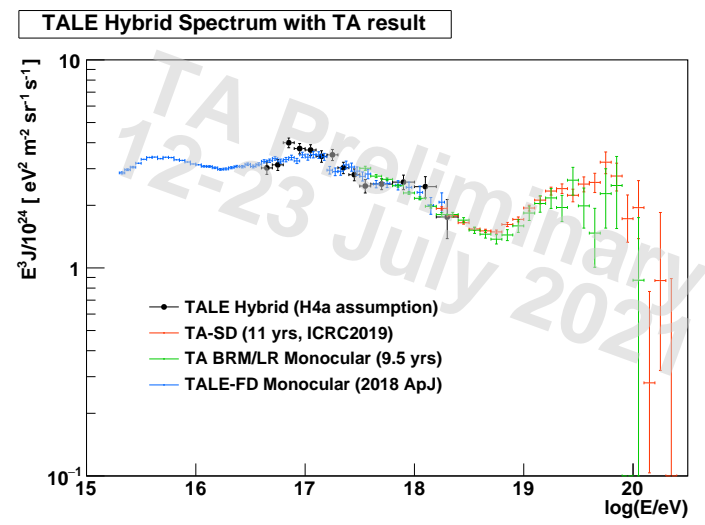

(b)

Figure 7: (a): Estimated exposure over the time period of 11/2017-02/2021. (b):The cosmic rays energy spectrum measured with TALE Hybrid detector. Here we use primary composition given by the H4a model. We also show measurements by TA using 9.5 yrs FDs at BRM and LR data [11], by 11 yrs TA-SD data [12] and 2 yrs TALE FD monocular data [3].

\section{Conclusion}

The total of 80 SDs were deployed and the TALE SD array started operation. In addition the observation with hybrid triggering system started in November 2018. We report on a first result of the preliminary cosmic ray composition and spectrum measured by TALE Hybrid detector. The obtained spectrum is consistent with our previous measurements. An examination of the mean $X_{\max }$ versus energy, shows a change in the $X_{\max }$ elongation rate at an energy $\sim 10^{17.1} \mathrm{eV}$.

\section{Acknowledgement}

The TALE SD production and the TALE hybrid operations are supported by the Japan Society for the Promotion of Science(JSPS) through Grants-in-Aid for Scientific Research (S) 15H05741 and 19H05607; by the joint research program of the Institute for Cosmic Ray Research (ICRR), The University of Tokyo. The experimental site became available through the cooperation of the Utah School and Institutional Trust Lands Administration (SITLA), U.S. Bureau of Land Management (BLM), and the U.S. Air Force. We appreciate the assistance of the State of Utah and Fillmore offices of the BLM in crafting the Plan of Development for the site. The people and the officials of Millard County, Utah have been a source of steadfast and warm support for our work which we greatly appreciate. We gratefully acknowledge the contribution from the technical staffs of our 
home institutions. The full acknowledgements are found in the contribution paper for the highlights of the Telescope Array experiments in this conference proceedings.

\section{References}

[1] J.H. Boyer, B.C. Knapp, E.J. Mannel and M. Seman, Nucl. Instrum. Meth. A 482 (2002) 457.

[2] Telescope Array collaboration, S. Ogio, PoS ICRC2019 (2019) 375.

[3] Telescope Array collaboration, R.U. Abbasi et al., Astrophys. J. 865 (2018) 74 [1803.01288].

[4] T.Z.AbuZayyad, Ph.D. thesis, University of Utah, 2000.

[5] T.K.Gaisser and A.M.Hillas, vol. Proceedings of 15th International Cosmic Ray Conference (Plovdiv, Bulgaria) 8, 1977.

[6] B.T. Stokes, R. Cady, D. Ivanov, J.N. Matthews and G.B. Thomson, Astropart. Phys. 35 (2012) 759 [1104.3182].

[7] S. Ostapchenko, Phys. Rev. D 83 (2011) 014018 [1010. 1869].

[8] Telescope Array collaboration, R.U. Abbasi et al., Astrophys. J. 858 (2018) 76 [1801.09784].

[9] T. Abu-Zayyad, K. Belov, D.J. Bird, J. Boyer, Z. Cao, M. Catanese et al., The Astrophysical Journal 557 (2001) 686.

[10] T.K. Gaisser, T. Stanev and S. Tilav, Front. Phys. (Beijing) 8 (2013) 748 [1303. 3565].

[11] G.D. Furlich, Ph.D. thesis, University of Utah, 2020.

[12] Telescope Array collaboration, D. Ivanov, PoS ICRC2019 (2020) 298. 


\section{Full Authors List: Telescope Array Collaboration}

R.U. Abbasi ${ }^{1,2}$, T. Abu-Zayyad ${ }^{1,2}$, M. Allen ${ }^{2}$, Y. Arai $^{3}$, R. Arimura ${ }^{3}$, E. Barcikowski ${ }^{2}$, J.W. Belz ${ }^{2}$, D.R. Bergman ${ }^{2}$, S.A. Blake ${ }^{2}$, I. Buckland ${ }^{2}$, R. Cady $^{2}$, B.G. Cheon ${ }^{4}$, J. Chiba ${ }^{5}$, M. Chikawa ${ }^{6}$, T. Fujii ${ }^{7}$, K. Fujisue ${ }^{6}$, K. Fujita ${ }^{3}$, R. Fujiwara ${ }^{3}$, M. Fukushima ${ }^{6}$, R. Fukushima ${ }^{3}$, G. Furlich ${ }^{2}$, R. Gonzalez ${ }^{2}$, W. Hanlon ${ }^{2}$, M. Hayashi ${ }^{8}$, N. Hayashida ${ }^{9}$, K. Hibino ${ }^{9}$, R. Higuchi ${ }^{6}$, K. Honda $^{10}$, D. Ikeda ${ }^{9}$, T. Inadomi ${ }^{11}$, N. Inoue ${ }^{12}$, T. Ishii ${ }^{10}$, H. Ito ${ }^{13}$, D. Ivanov ${ }^{2}$, H. Iwakura ${ }^{11}$, A. Iwasaki ${ }^{3}$, H.M. Jeong ${ }^{14}$, S. Jeong ${ }^{14}$, C.C.H. Jui ${ }^{2}$, K. Kadota ${ }^{15}$, F. Kakimoto ${ }^{9}$, O. Kalashev ${ }^{16}$, K. Kasahara ${ }^{17}$, S. Kasami ${ }^{18}$, H. Kawai ${ }^{19}$, S. Kawakami ${ }^{3}$, S. Kawana ${ }^{12}$, K. Kawata ${ }^{6}$, I. Kharuk ${ }^{16}$, E. Kido ${ }^{13}$, H.B. Kim ${ }^{4}$, J.H. Kim ${ }^{2}$, J.H. Kim ${ }^{2}$, M.H. Kim ${ }^{14}$, S.W. Kim ${ }^{14}$, Y. Kimura ${ }^{3}$, S. Kishigami ${ }^{3}$, Y. Kubota ${ }^{11}$, S. Kurisu ${ }^{11}$, V. Kuzmin ${ }^{16^{\dagger}}$, M. Kuznetsov ${ }^{16,20}$, Y.J. Kwon ${ }^{21}$, K.H. Lee ${ }^{14}$, B. Lubsandorzhiev ${ }^{16}$, J.P. Lundquist ${ }^{2,22}$, K. Machida ${ }^{10}$, H. Matsumiya ${ }^{3}$, T. Matsuyama ${ }^{3}$, J.N. Matthews ${ }^{2}$, R. Mayta ${ }^{3}$, M. Minamino ${ }^{3}$, K. Mukai ${ }^{10}$, I. Myers ${ }^{2}$, S. Nagataki ${ }^{13}$, K. Nakai ${ }^{3}$, R. Nakamura ${ }^{11}$, T. Nakamura ${ }^{23}$, T. Nakamura ${ }^{11}$, Y. Nakamura ${ }^{11}$, A. Nakazawa ${ }^{11}$, E. Nishio ${ }^{18}$, T. Nonaka ${ }^{6}$, H. Oda ${ }^{3}$, S. Ogio ${ }^{3,24}$, M. Ohnishi ${ }^{6}$, H. Ohoka ${ }^{6}$, Y. Oku ${ }^{18}$, T. Okuda ${ }^{25}$, Y. Omura ${ }^{3}$, M. Ono ${ }^{13}$, R. Onogi ${ }^{3}$, A. Oshima ${ }^{3}$, S. Ozawa ${ }^{26}$, I.H. Park ${ }^{14}$, M. Potts ${ }^{2}$, M.S. Pshirkov ${ }^{16,27}$, J. Remington ${ }^{2}$, D.C. Rodriguez ${ }^{2}$, G.I. Rubtsov ${ }^{16}$, D. Ryu ${ }^{28}$, H. Sagawa ${ }^{6}$, R. Sahara ${ }^{3}$, Y. Saito ${ }^{11}$, N. Sakaki ${ }^{6}$, T. Sako ${ }^{6}$, N. Sakurai ${ }^{3}$, K. Sano ${ }^{11}$, K. Sato ${ }^{3}$, T. Seki ${ }^{11}$, K. Sekino ${ }^{6}$, P.D. Shah ${ }^{2}$, Y. Shibasaki ${ }^{11}$, F. Shibata ${ }^{10}$, N. Shibata ${ }^{18}$, T. Shibata ${ }^{6}$, H. Shimodaira ${ }^{6}$, B.K. Shin ${ }^{28}$, H.S. Shin ${ }^{6}$, D. Shinto ${ }^{18}$, J.D. Smith ${ }^{2}$, P. Sokolsky ${ }^{2}$, N. Sone ${ }^{11}$, B.T. Stokes ${ }^{2}$, T.A. Stroman ${ }^{2}$, Y. Takagi ${ }^{3}$,

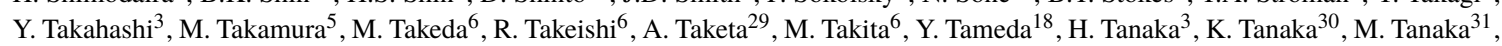
Y. Tanoue ${ }^{3}$, S.B. Thomas ${ }^{2}$, G.B. Thomson ${ }^{2}$, P. Tinyakov ${ }^{16,20}$, I. Tkachev ${ }^{16}$, H. Tokuno ${ }^{32}$, T. Tomida ${ }^{11}$, S. Troitsky ${ }^{16}$, R. Tsuda ${ }^{3}$, Y. Tsunesada ${ }^{3,24}$, Y. Uchihori ${ }^{33}$, S. Udo ${ }^{9}$, T. Uehama ${ }^{11}$, F. Urban ${ }^{34}$, T. Wong ${ }^{2}$, K. Yada $^{6}$, M. Yamamoto ${ }^{11}$, K. Yamazaki ${ }^{9}$, J. Yang ${ }^{35}$, K. Yashiro ${ }^{5}$, F. Yoshida ${ }^{18}$, Y. Yoshioka ${ }^{11}$, Y. Zhezher ${ }^{6,16}$, and Z. Zundel ${ }^{2}$

${ }^{1}$ Department of Physics, Loyola University Chicago, Chicago, Illinois, USA

${ }^{2}$ High Energy Astrophysics Institute and Department of Physics and Astronomy, University of Utah, Salt Lake City, Utah, USA

${ }^{3}$ Graduate School of Science, Osaka City University, Osaka, Osaka, Japan

${ }^{4}$ Department of Physics and The Research Institute of Natural Science, Hanyang University, Seongdong-gu, Seoul, Korea

${ }^{5}$ Department of Physics, Tokyo University of Science, Noda, Chiba, Japan

${ }^{6}$ Institute for Cosmic Ray Research, University of Tokyo, Kashiwa, Chiba, Japan

${ }^{7}$ The Hakubi Center for Advanced Research and Graduate School of Science, Kyoto University, Kitashirakawa-Oiwakecho, Sakyo-ku, Kyoto, Japan

${ }^{8}$ Information Engineering Graduate School of Science and Technology, Shinshu University, Nagano, Nagano, Japan

${ }^{9}$ Faculty of Engineering, Kanagawa University, Yokohama, Kanagawa, Japan

${ }^{10}$ Interdisciplinary Graduate School of Medicine and Engineering, University of Yamanashi, Kofu, Yamanashi, Japan

${ }^{11}$ Academic Assembly School of Science and Technology Institute of Engineering, Shinshu University, Nagano, Nagano, Japan

${ }^{12}$ The Graduate School of Science and Engineering, Saitama University, Saitama, Saitama, Japan

${ }^{13}$ Astrophysical Big Bang Laboratory, RIKEN, Wako, Saitama, Japan

${ }^{14}$ Department of Physics, SungKyunKwan University, Jang-an-gu, Suwon, Korea

${ }^{15}$ Department of Physics, Tokyo City University, Setagaya-ku, Tokyo, Japan

${ }^{16}$ Institute for Nuclear Research of the Russian Academy of Sciences, Moscow, Russia

${ }^{17}$ Faculty of Systems Engineering and Science, Shibaura Institute of Technology, Minato-ku, Tokyo, Japan

${ }^{18}$ Department of Engineering Science, Faculty of Engineering, Osaka Electro-Communication University, Neyagawa-shi, Osaka, Japan

${ }^{19}$ Department of Physics, Chiba University, Chiba, Chiba, Japan

${ }^{20}$ Service de Physique Théorique, Université Libre de Bruxelles, Brussels, Belgium

${ }^{21}$ Department of Physics, Yonsei University, Seodaemun-gu, Seoul, Korea

${ }^{22}$ Center for Astrophysics and Cosmology, University of Nova Gorica, Nova Gorica, Slovenia

${ }^{23}$ Faculty of Science, Kochi University, Kochi, Kochi, Japan

${ }^{24}$ Nambu Yoichiro Institute of Theoretical and Experimental Physics, Osaka City University, Osaka, Osaka, Japan

${ }^{25}$ Department of Physical Sciences, Ritsumeikan University, Kusatsu, Shiga, Japan

${ }^{26}$ Quantum ICT Advanced Development Center, National Institute for Information and Communications Technology, Koganei, Tokyo, Japan

27 Sternberg Astronomical Institute, Moscow M.V. Lomonosov State University, Moscow, Russia

${ }^{28}$ Department of Physics, School of Natural Sciences, Ulsan National Institute of Science and Technology, UNIST-gil, Ulsan, Korea

${ }^{29}$ Earthquake Research Institute, University of Tokyo, Bunkyo-ku, Tokyo, Japan

${ }^{30}$ Graduate School of Information Sciences, Hiroshima City University, Hiroshima, Hiroshima, Japan

${ }^{31}$ Institute of Particle and Nuclear Studies, KEK, Tsukuba, Ibaraki, Japan

32 Graduate School of Science and Engineering, Tokyo Institute of Technology, Meguro, Tokyo, Japan

33 Department of Research Planning and Promotion, Quantum Medical Science Directorate, National Institutes for Quantum and Radiological Science and Technology, Chiba, Chiba, Japan

${ }^{34}$ CEICO, Institute of Physics, Czech Academy of Sciences, Prague, Czech Republic

${ }^{35}$ Department of Physics and Institute for the Early Universe, Ewha Womans University, Seodaaemun-gu, Seoul, Korea

\footnotetext{
${ }^{\dagger}$ Deceased
} 